\title{
Consideration on the Accuracy of Watch for Physical Activity Tracker
}

Tagliente Irene ${ }^{1-3^{*}}$, Trieste Leopoldo ${ }^{4}$, Petrassi Valerio ${ }^{3}$, De Balsi Vincenzo Mario ${ }^{3}$ and Bella Sergio ${ }^{5}$

${ }^{1}$ Scientific Direction, Research Area of Clinical-Healthcare and Management Innovations, Pediatric Hospital Bambino Gesù, Rome, Italy

${ }^{2}$ Department of Human Social and Health Sciences, University of Cassino and Southern Lazio, Cassino-Italy

${ }^{3}$ Department of Information Engineering, Electronics and Telecommunications, Faculty of Engineer, University "La Sapienza", Rome-Italy

${ }^{4}$ Institute of Management, Scuola Superiore Sant'Anna, Pisa, Italy

${ }^{5}$ Unit of Cystic Fibrosis, Department of Pediatric Medicine-Pediatric Hospital Bambino Gesù, Rome, Italy

"Corresponding author: Irene Tagliente, Clinical-Healthcare and Management Innovations Research Area, Bambino Gesù Children's Hospital, Piazza Sant'Onofrio 1 , 00146, Roma, Italy Tel:+393492310849; E-mail: Irene.tagliente@opbg.net

Received date: May 26, 2016, Accepted date: February 18, 2017, Published date: February 24, 2017

Copyright: ( 2017 Irene T et al. This is an open-access article distributed under the terms of the Creative Commons Attribution License, which permits unrestricted use, distribution, and reproduction in any medium, provided the original author and source are credited.

\section{Abstract}

Background: Obesity is one of the biggest drivers of preventable chronic diseases and healthcare costs in Worldwide. The most difficult aspect to take under consideration on the developing algorithms able to value the single session of kcal consumption in a single session of training are the hormonal factors.

Objective: In this study we done a preliminary study on the comparison of two different watch (that provide an estimate of kilocalories consumed with and without heart rate monitor) with an indirect calorimetry device. The aim of this study was to understand if the watch could be considered for health use and the variable that can influence the valuation accuracy of the kcal consumption during a training session on gym.

Methods: In this preliminary study we analyzed the indicators that influence the daily energy expenditure and possible device able to track it, considering possible limits of application. Therefore, we analyzed the critical issue emerged to track each indicator.

Results: From this preliminary study was possible to show that the accuracy of the device could have important difference case by case. More information have to be done to the customer to understand the accuracy of the different device able to detect the kcal consumption because it can modify the diet of not professional people. We had problem with the synchronization of health rate monitor and the watch with Bluetooth mode in room with computer or a lot of IT equipment.

Discussion: This preliminary study showed that could be more vital signals to take under consideration developing algorithm for the daily kcal consumption. More study are need to understand way we have a difference of accuracy during a reliable test in healthy volunteers. Metabolism aspect could be taken under consideration starting from consideration related on weight and high of subject.

Conclusion: The conclusion was not permitted to have the algorithm used to detect the kcal consumption so we did some preliminary test. From as analysis we show a not constant accuracy for the watch 1 and watch 2 vs. the indirect calorimetry. This suggest that some more variable have to be taken under consideration in developing of device, as suggested from the literature.

Keywords: Physical activity; Motor activities; Psychomotor performance; Energy expenditure; Energy metabolisms; Instruments and Apparatus; Telemedicine; Mobile health; Telehealth

\section{Abbreviation \\ EE: Energy Expenditure; PA: Physical Activity; QOL: Quality of Life: QALY: Quality Adjusted Life Year; OSA: Obese Sleep Apnea; DEE: Daily Energetic Expense of an individual; ET: Endogenous Thermogenesis; EEPA: Energetic Expense linked to the Physical Activity; BMR: Basal Metabolic Rate}

\section{Introduction}

Obesity is one of the biggest drivers of preventable chronic diseases and healthcare costs in Worldwide. Different prevention activities are suggested. Starting from accurate evaluation of personal daily energy expenditure (EE) it is possible to plan a correct and personalized diets, as well as programming physical activity (PA), being the last one of the most important factors influencing health.

Physical inactivity is one of the most important public health problems of the 21 st century $[1,2]$. Rates of physical inactivity, that are rapidly growing worldwide, have attracted the attention of the international healthcare community $[1,3-5]$ and the proportion of physical exercise [2-5]. 
Physical inactivity is associated with the increased risk of several chronic diseases; at the same time incidence of the disease with the high social burden decrease with a good physical activity. Also, some economic studies has proved that physical activity is cost-effective, offering good value for money, with relevant gains in both survival and health-related quality of life (QOL), and with reasonable cost-per quality adjusted life year (QALY) and cost per-life-year ratios [6]. In the last years is growing the interest of the company on the developing of PA tracker. In this context, and thanks to advances in communications technology, mobile devices have emerged as an important tool for disease prevention and intervention affecting health habits and disease evolution [6].

Therefore, the different health applications need different level of accuracy. We aspect a high accuracy in device used for heath or training application, then for not professional use. Also, related to the PA will be useful, for professional use, the simultaneous analysis of vital signals, first the health rate [7].

In daily life, walking is one of the most energy consuming activity. Many researchers proposed and adopted GPS and 3-axis accelerometers for location tracking and activity monitoring, for assessment the related energy expenditure. However, in the case of walking on a slope, this method fails [8].

The proposed solution can estimate energy expenditure in condition of static position or slow motion, but it is less accurate and strongly influenced by the environment with different level of accuracy with faster movement e.g., elevation in slope and jumping usually increase the level of energy expenditure [8].

The Energy Expenditure of the human body is reduced during sleep. This change is influenced by circadian rhythm, changes in body temperature, and reduction in muscle activity. Development of adaptive thermogenesis has been proposed to explain the repetitive failure of weight reduction programs. But its exact role in obese with Sleep Apnea (OSA) patients is not clearly demonstrated $[9,10]$.

In this contest, multi-sensor, accelerometer-based devices have the potential to improve the estimation of $\mathrm{EE}$ in people with movement disorders [11]. Predictive equations, which are usually based on the body weight, height, age and gender, are used to estimate the EE in clinical settings. Many studies have developed and validated those predictive equations [11-13].

However, the best equation to estimate $\mathrm{EE}$ is difficult to establish because of conflicting results in different conditions and people characteristics [14].

The most difficult aspect to take under consideration on the developing algorithms able to value the single session of k.cal consumption in a single session of training are the hormonal factor.

In this study, we done a preliminary study on the comparison of two different watch (that provide an estimate of kilocalories consumed with and without heart rate monitor) with an indirect calorimetry device.
The aims of this study were to understand if the watch could be considered for health use and the variable that can influence the valuation accuracy of the kcal consumption during a training session on gym.

In this way, at the core of the telemonitoring lifestyle of athletes, chronic patients or old people, there is the technical problem of measuring daily energy expenditure, as we show in previous study [7], modulated in consideration of daily PA.

\section{Materials and Methods}

The study was focused the attention on the analysis of factors that can be considered as indicators for the daily physical activity track. In this way, at the core of the telemonitoring lifestyle of athletes, chronic patients or old people, there is the technical problem of measuring daily energy expenditure, modulated in consideration of daily PA.

In this study, we analyzed the indicators that influence the daily energy expenditure and possible device able to track it, considering possible limits of application. Therefore, we analyzed the critical issue emerged to track each indicator.

The literature review was carried out using the major search engines, as well as sectorial and generalist electronic databases (PubMed, ADA, Google Scholar, Google Patents, CDA, IEEE Journals).

In this preliminary study, we make the acquisition of data on the Motor. In this study, we also tested two devices available in the market to value the usability and accuracy of measurement. We selected two different watch, with expected high level of accuracy, with not public kcal algorithm.

Comparison was based on the analysis of the different kcal calculated from the watch during acquisition. Tests on data acquisition were carried out at the Sports Sciences Laboratories of University of Cassino and Southern Lazio (Cassino, Italy), in collaboration with the Electrical and Information Engineering Department of that University, and using the K4 Cosmed as a gold standard for measuring indirect calorimetry.

The analysis of the data was carried out at the Clinical-Healthcare and Management Innovations Research Area of the Bambino Gesù Children's Hospital (Rome, Italy), and Faculty of Engineer at University La Sapienza (Rome, Italy).

All information acquired was mash with the technical skill in Telemedicine of the works team, and discussed focusing the attention on the operative device applications.

We enrolled student's volunteer of Department of Human Social and Health Sciences, - University of Cassino and Southern Lazio, Cassino-Italy [Table 1].

We equipped each Subject with: 2 watch with its heart rate monitor, K4 Cosmed mask in Telemetry function and a temperature data logger.

\begin{tabular}{|l|l|l|l|l|l|l|}
\hline No of Subject & Man & Female & Age & Weight (Kg) & High \\
\hline 6 & 4 & 2 & $25 \pm 3$ & $50-87$ & $160<\mathrm{x}<185$ \\
\hline
\end{tabular}

Table 1: Characteristics of voluntary patients. 
The acquisition protocol was the same for all volunteer:

-5 minutes of relax in a room with $25^{\circ} \mathrm{C}$ and $40 \%$ of Humidity to acquire the $\mathrm{VO}_{2}$ max predicted (conditions required from one of the two watch)

-3 minutes of heating on a cycle ergometer

-18 minutes of cycling with load proportionate to the weight of the subject

-5 minutes of recovery.

The data was stored in real time, marking the data read on the display and/or downloaded with USB on the dedicated software. Therefore all data was exported on .csv file and analyzed of .exl format. In this was possible, for all subject, to update the watches with the individual parameter as: birth date, sex, height and weight.

The data was analyzed to Faculty of Engineer at University La Sapienza (Rome, Italy), where was take under consideration the coefficient (Table 2) of equivalent surface dimensions (Ad).

Also, we make some temperature acquisition before and during a test session on tapis roulant on 14 volunteer to analyze the difference before, during and after the test.

The test session protocol was the same for all volunteer:

-5 minutes of relax in a room with $25^{\circ} \mathrm{C}$ and $40 \%$ of Humidity to acquire the $\mathrm{VO}_{2}$ max predicted (conditions required from one watch)

-10 minutes of tapis roulant with level 10 of speed and $2 \%$ slope

-5 minutes of recovery.

For the test on the tapis roulant we take the temperature value with a digital thermometer every 5 minutes.

\section{Result}

The international literature converges to the Daily Energetic Expense (DEE) as then energy organism consumes daily as indicator that collect the most useful information on daily activity. This indicator sums the Endogenous Thermogenesis (ET), the Energetic Expense related to the Physical Activity (EEPA), and the Basal Metabolic Rate (BMR) i.e., the minimal energy that an organism needs to be alive. It constitutes from 60 to $70 \%$ of the EEPA in the majority of the sedentary adults, while, in very active individuals it is of approximately $50 \%$. It changes depending on the body composition, especially on the body lean mass [12,15]. Energy metabolism and food intake are complex processes that involve homeostatic mechanisms driven by hypothalamic network signaling pathways [11].

Predictive equations, which are usually based on the body weight, height, age and gender, are used to estimate the EE in clinical settings. Many studies have developed and validated those predictive equations [11-13]. However, the best equation to estimate $\mathrm{EE}$ is difficult to establish because of conflicting results in different conditions and people characteristics [14].

As illustrated by Jesus et al. (2015) [13] the growing number of equations makes difficult the selection of specific equations that will work well in different contexts. Some equations include VO2 max as fundamental indicator but at the same time in not enough clear the right way to calculate it out of laboratory or hospital. We show that in the recent years some study starting to take into consideration the impact of thermoregulation looking for new ET prediction model [16].
The daily EE is estimated with a combination of BMR and energy expenditure during physical activity.

Some devices have integrated additional sensors and instruments like thermometers, parameters they assess are not still included into the EE algorithm.

We compared the kcal calculated simultaneous from each watch during the test session and the kcal calculated from the K4 in the same session.

From this preliminary study was possible to show that the accuracy of the device could have important difference case by case (Tables 2-4).

\begin{tabular}{|l|}
\hline $\mathbf{A d}=\mathbf{0 , 2 0 2} \mathbf{W}^{\wedge} \mathbf{0 , 4 2 5} \mathbf{H}^{\wedge} \mathbf{0} 0,725$ \\
\hline $\mathrm{W}=$ Weight \\
$\mathrm{H}=$ High
\end{tabular}

Table 2: Dubois equation.

\begin{tabular}{|l|l|l|l|}
\hline & Gold Standard & Watch 1 & Watch 2 \\
\hline Average & 5.45 & 5.74 & 5.72 \\
\hline Variance & 0.206 & 0.401 & 1.687 \\
\hline Average / Variance & 26.44 & 14.31 & 3.39 \\
\hline
\end{tabular}

Table 3: Average and variance analysis.

\begin{tabular}{|l|l|}
\hline $\begin{array}{l}\text { Percentage of difference from } \\
\text { Gold Standard VS Watch } \\
\text { considering AD }\end{array}$ & $\begin{array}{l}\text { Percentage of difference from Gold } \\
\text { Standard VS Watch 2 considering AD }\end{array}$ \\
\hline $28 \%$ & $N^{\circ}$ \\
\hline $3 \%$ & $22 \%$ \\
\hline $5 \%$ & $N A^{\circ}$ \\
\hline $4 \%$ & $-8 \%$ \\
\hline $1 \%$ & $N A^{\circ}$ \\
\hline$-7 \%$ & $-17 \%$ \\
\hline data not available & \\
\hline
\end{tabular}

Table 4: Percentage of difference from the Gold Standard.

From this analysis, we show a not constant accuracy in the range of $+-3 \%$, as expected. From this preliminary study, we show unexpected inaccuracy $>20 \%$.

Regards of thermoregulation, we show (Figure 1), as expected an increase of temperature with health rate, until the brought into operation of thermoregulatory mechanisms and thus the appearance of sweat. Also, we analyzed the temperature data acquired in one other set of volunteers.

We show the same trend, so an increase of $t$ increase of temperature with health rate, until the brought into operation of thermoregulatory mechanisms and thus the appearance of sweat. We make a t-test of the data, the difference of temperature before, during and after the session test, was not significant $p>0,05$. Therefore, the kcal acquisition without 
heart rate monitor was lower than $20 \%$ in the watch 1 and not permitted by the watch 2 .

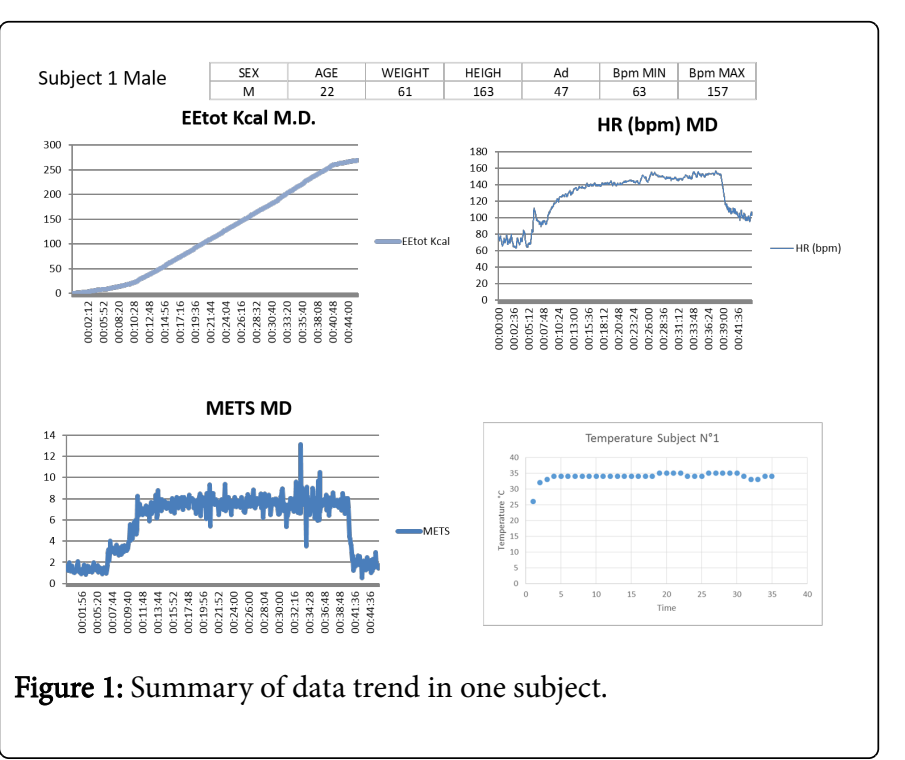

\section{Conclusion}

The individual energy expenditure is significantly influenced by the daily PA. The constant monitoring, of the balance between the kcal intake and the individual $\mathrm{EE}$, could be a good way to have a completed clinical assessment of patients and in athlete training program. Also, the analysis of individual $\mathrm{EE}$ is important to make a right diet for patients with chronic disease. In this study, we focused the attention on the factors that can influence the individual EE starting to the analysis of metabolic process.

Thermoregulation it is an important body activity, but there is not enough evidence on the correlation between $\mathrm{EE}$ and thermogenesis actions.

In this preliminary study, we analyzed two different watch declared able to detect the kcal consumption during activity with health rate monitor. Was not permitted to have the algorithm used to detect the kcal consumption so we did some preliminary test.

From as analysis we show a not constant accuracy for the watch 1 and watch $2 \mathrm{Vs}$ the indirect calorimetry.

This suggests that some more variable must be taken under consideration in developing of device, as suggested from the literature.

Also, we analyzed the possible impact of the thermoregulation but was not possible to detect a significance difference of the temperature before, during and after the session test. The thermoregulation effect was difficult to detect because of the influence of ambient temperature. This short overview suggests the importance of developing new wearable devices, also for the clinical practice [17]. Wearable device can be introduced to encourage the prevention actions, selfmanagement of disease and telemedicine assistance.

More information must be done to the customer to understand the accuracy of the different device able to detect the kcal consumption because it can modify the diet of not professional people.
We had problem with the synchronization of health rate monitor and the watch with Bluetooth mode in room with computer or a lot of IT equipment.

\section{Discussion and Future Work}

A right analysis of the PA could be helpful for different applications. Many studies explain the good impact of applications of Telemedicine on public health $[18,19]$.

Today, the devices available for measuring EE accurately require specialized professionals and sophisticated methodologies that are costly and difficult to conduct. With respect to thermoregulation indicators, the analysis of variance on the body temperature in different climatic and motion condition must be taken in more consideration.

This preliminary study showed that could be more vital signals to take under consideration developing algorithm for the daily kcal consumption. More study is need to understand way we have a difference of accuracy during a reliable test in healthy volunteers. Metabolism aspect could be taken under consideration starting from consideration related on weight and high of subject.

The body temperature could be important variable but the right acquisition could be difficult in conventional conditions and so also in daily activity.

Different sensors connected with mobile devices could be important tools to consider all body variations, also for disease prevention and keep track of intervention, affecting health state evolution and costs. In this way, new mobile device applications, for PA track, are also helpful for Telehealth to have a punctual telemonitoring of exercise regime of patients, especially oldest and/or with chronic disease is mandatory, specially to specialize dietary intake [7].

In the developing of wearable $\mathrm{t}$-shirt will be important to have difference size to have good adherence of it, and so low acquisition and interference errors.

The Bluetooth mode must be developed considering the possibility of need the synchronization of the device component also in room with a lot of computer as office, for daily use.

\section{References}

1. Benzo RP, Chang CC, Farrell MH, Kaplan R, Ries A, et al. (2010) Physical activity, health status and risk of hospitalization in patients with severe chronic obstructive pulmonary disease. Respiration 80:10-18.

2. Barnes PM and Schoenborn CA (2012) Trends in adults receiving a recommendation for exercise or other physical activity from a physician or other health professional. NCHS Data Brief 1-8.

3. Fernandes RA, Coelho ESMJ (2015) Possible underestimation by sports medicine of the effects of early physical exercise practice on the prevention of diseases in adulthood. Curr Diabetes Rev 11:201-205.

4. Kahlmeier STM, Wijnhoven W (2015) National physical activity recommendations: systematic overview and analysis of the situation in European countries. BMC Public Health 15:133.

5. Blair SN (2009) Physical inactivity: the biggest public health problem of the 21st century. Br J Sports Med. 43:1-2.

6. Roux L, Pratt M, Tengs TO, Yore MM, Yanagawa TL, et al. (2008) Cost effectiveness of community-based physical activity interventions. Am J Prev Med 35:578-588.

7. Tagliente I, Solvoll T, Trieste L, De Cecco CN, Murgia F, et al. (2016) Which indicators for measuring the daily physical activity? An overview 
Citation: Irene T, Leopoldo T, Valerio P, Vincenzo Mario De B, Sergio B (2017) Consideration on the Accuracy of Watch for Physical Activity Tracker. Gen Med (Los Angeles) 5: 284. doi:10.4172/2327-5146.1000284

Page 5 of 5

on the challenges and technology limits for Telehealth applications. Technol Health Care 24:665-672.

8. Hwan Jung,Young-Dong Lee, Do-Un Jeong (2011) A novel method for energy expenditure using multisensor based activity monitoring. Computer Sciences and Convergence Information Technology (ICCIT) 103-106.

9. Bamberga M, Rizzi M, Gadaleta F, Grechi A, Baiardini R, et al. (2015) Relationship between energy expenditure, physical activity and weight loss during CPAP treatment in obese OSA subjects. Respir Med. 109:540-545.

10. Hins J, Sériès F, Alméras N, Tremblay A (2006) Relationship between severity of nocturnal desaturation and adaptive thermogenesis: preliminary data of apneic patients tested in a whole-body indirect calorimetry chamber. Int J Obes (Lond) 30:574-577.

11. Ryan JM, Walsh M, Gormley J (2014) A comparison of three accelerometry-based devices for estimating energy expenditure in adults and children with cerebral palsy. J Neuroeng Rehabil 11:116.

12. Blasco Redondo R (2015) Resting energy expenditure; assessment methods and applications. Nutr Hosp. 3:245-254

13. Jésus P, Achamrah N, Grigioni S, Charles J, Rimbert A, et al. (2015) Validity of predictive equations for resting energy expenditure according to the body mass index in a population of 1726 patients followed in a Nutrition Unit. Clin Nutr. 34:529-535.

14. Cutillo L, Pizziconi C, Tozzi AE, Verrillo E, Testa MB, et al. (2014) Predicted and Measured Resting Energy Expenditure in Children with Spinal Muscular Atrophy 2. J Pediatr 164:1228-1230.

15. Cunningham JJ (1980) A reanalysis of the factors influencing basal metabolic rate in normal adults. Am J Clin Nutr 33:2372-2374.

16. Ocobock C (2016) The allocation and interaction model: A new model for predicting total energy expenditure of highly active humans in natural environments. Am J Hum Biol. 28:372-380.

17. Arsand E, Muzny M, Bradway M, Muzik J, Hartvigsen G (2015) Performance of the first combined smartwatch and smartphone diabetes diary application study. J Diabetes Sci Technol 9:556-563.

18. Schiaffini R, Tagliente I, Carducci C, Ullmann N, Ciampalini P, et al. (2016) Impact of long-term use of eHealth systems in adolescents with type 1 diabetes treated with sensor-augmented pump therapy. J Telemed Telecare 22:277-281.

19. Muntaner A, Vidal-Conti J, Palou P (2016) Increasing physical activity through mobile device interventions: A systematic review. Health Informatics J 22: 451-469. 